\title{
EXPERIMENTAL VISION SYSTEM SETUP BASED ON THE SERIAL CONFIGURATION INTERFACE
}

\author{
Martin Varga \\ Technical University of Kosice, Faculty of Mechanical Engineering, Letna 9, Kosice, Slovak Republic, EU, \\ martin.varga.2@tuke.sk (corresponding author) \\ Marek Vagaš \\ Technical University of Kosice, Faculty of Mechanical Engineering, Letna 9, Kosice, Slovak Republic, EU, \\ marek.vagas@tuke.sk
}

\begin{abstract}
Keywords: data transfer, automation, communication, camera system
Abstract: Successful SME companies see indisputable advantages in implementing and image processing of vision systems. The proposed technical solution described in this article points to the need for deploying the advanced functions of vision systems to achieve the relevant computing power in the form of CCD cameras. It is expected to be used in a real-life automated consisting of, among others, a standard industrial robot. Here the starting point of the manufacturing process is the automatic selection and detection of arbitrarily oriented objects. In addition to the above, the article aims to point out the current progress in this area and draw attention to comparative methods within advanced camera systems. The resulting topics will gradually introduce us to the field of advanced image processing, where the use of functions such as object reconstruction or property estimation is assumed.
\end{abstract}

\section{Introduction}

Vision systems are, in general, systems that use a camera and various image analysing software to extract useful information from an image. These systems are flexible, highly adaptive, and can be used for a broad range of tasks like tracking moving objects [1], vibration, displacement and strain analysis [2], object position and orientation detection in manufacturing [3], etc.

Advantages that come from the implementation of vision systems in automated workplaces together with their impact cannot be disputed. This article is aimed at obtaining some chosen object characteristic, mainly the 3D coordinate information, using a vision system. The proposed solution (based on RS232C communication interface) can be considered as a relevant starting point to picture capturing, advanced sensing and object data processing followed by data processing with the help of a powerful pc workstation [4].

Verified technical solution - an experimental stand equipped with CCD cameras designed for easy implementation into a real manufacturing workplace that is containing a SCARA robotic arm. Its potential purpose is the automated selection and recognition of objects that arrive from oriented vibratory trays. Beside this, reliable automation of manufacturing processes can be reached by interconnection with an advanced control level (ERP, MAS etc...) [5]. Besides the experimental data obtained by using this stand, students, teachers and potential cooperating companies can acquire higher levels of skill and experience in the field of vision systems. TCP / IP communication protocol will be implemented during future research.
Apart from the above-mentioned properties, it is the aim of our experiments to apply comparative methods in combination with vision systems. [6]. Future research will be focused on processing of an object and determination of its properties via sensing from different angles and sides. As a next step, the captured information can be processed by dedicated software.

\section{Communication}

Data transfer between the robotic arm and the vision system is realized via RS232C communication channel. A both way communication with the use of serial channel during the process is assumed. [7]. The configuration process is followed by a cold start-up of the control system of the robotic arm.

Serial interface must be configured in respect to the operating system in a way suitable for transmission with the use of CREAD / CWRITE command, see Table 1, 2, and 3. Serial communication can be usually configured through CREAD / CWRITE according to the followed figure, see figure 1 .

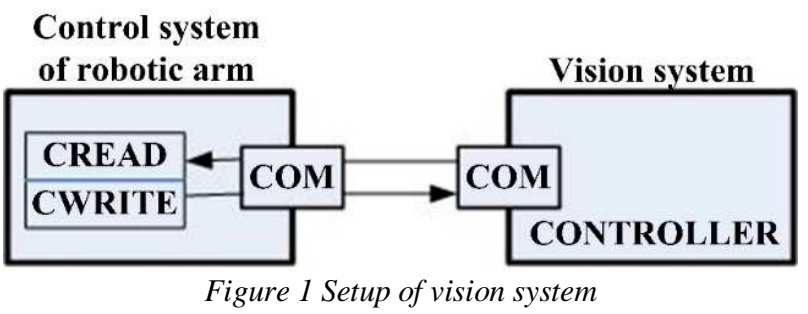
of robotic arm

Figure 1 Setup of vision system

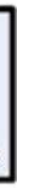


Table 1. Configuration of serial interface

\begin{tabular}{lll} 
1. & [COM3] & \\
\hline 2. & $\begin{array}{l}\text { BAUD }= \\
9600\end{array}$ & $\begin{array}{l}; 110,150,300,600,1200, \\
2400,4800,9600,19200,\end{array}$ \\
\hline 3. & $\begin{array}{l}\text { CHAR_LEN } \\
=8\end{array}$ & $; 7,8$ \\
\hline 4. & $\begin{array}{l}\text { STOP_BIT } \\
=1\end{array}$ & $; 1,2$ \\
\hline 5. & PARITY =2 & $\begin{array}{l}; \mathrm{EVEN}=2, \mathrm{ODD}=1, \\
\text { NONE }=0\end{array}$ \\
\hline 6. & PROC $=1$ & $\begin{array}{l}; 3964 \mathrm{R}=1, \mathrm{SRVT}=2, \\
\mathrm{WTC}=3, \mathrm{XON} / \mathrm{XOFF}=4\end{array}$
\end{tabular}

Table 2. Configuration of transmit protocol $3964 R$ that is used for the data transfer

\begin{tabular}{|c|c|c|}
\hline 1. & [3964R] & \\
\hline 2. & $\begin{array}{l}\text { CHAR_TIMEOUT = } \\
500\end{array}$ & $\begin{array}{l}\text {;msec, max. } \\
\text { interval between } \\
\text { two symbols }\end{array}$ \\
\hline 3. & $\begin{array}{l}\text { QUITT_TIMEOUT } \\
=500\end{array}$ & $\begin{array}{l}\text {;msec, } \max \text {. } \\
\text { waiting time at } \\
\text { control system of } \\
\text { robotic arm - to } \\
\text { symbol DLE }\end{array}$ \\
\hline 4. & $\begin{array}{l}\text { TRANS_TIMEOUT } \\
=500\end{array}$ & ;msec, \\
\hline 5. & $\begin{array}{l}\text { MAX_TX_BUFFER } \\
=2\end{array}$ & $\begin{array}{l}; 1 . .5, \text { max. value of } \\
\text { cache output }\end{array}$ \\
\hline 6. & $\begin{array}{l}\text { MAX_RX_BUFFER } \\
=10\end{array}$ & $\begin{array}{l}; 1 . .20, \text { max. value } \\
\text { of cache input }\end{array}$ \\
\hline 7. & $\begin{array}{l}\text { SIZE_RX_BUFFER } \\
=1 . .2048\end{array}$ & $\begin{array}{l}\text {;dimension of } \\
\text { receiving memory } \\
\text { input (in bytes) }\end{array}$ \\
\hline 8. & $\begin{array}{l}\text { PROTOCOL_PRIOR } \\
=1\end{array}$ & $\begin{array}{l}; \mathrm{HIGH}=1, \mathrm{LOW} \\
=0, \text { priority }\end{array}$ \\
\hline
\end{tabular}

Table 3. An example of successful communication between both systems (vision system and control system of robotic arm)

\begin{tabular}{cl}
\hline 1. & DEFDAT SEND \\
\hline 2. & ;DECLARATION \\
\hline 3. & INT HANDLE \\
\hline 4. & DECL STATE_T SW_T, SC_T \\
\hline 5. & DECL MODUS_T, MW_T \\
\hline 6. & ENDDAT \\
\hline 7. & DEF SEND () \\
\hline 8. & ;INITIALIZATION \\
\hline 9. & MW_T $=$ \#SYNC \\
\hline 10. & ;NSTRUCTION \\
\hline 11. & OPEN_P( $)$ \\
\hline 12. & WRITE() \\
\hline 13. & CLOSE_P() \\
\hline 14. & END
\end{tabular}

\section{Experimental testing - Setup}

Main aim of the experiment on the vision system OMRON F150-3 is to experimentally verify its declared properties, in-build functions and fields of use, especially the task of determining chosen object characteristic. Through this system it is possible to determine an observed object position in a working envelope, followed by the determination of coordinates of the centre of gravity $(x, y$, $\mathrm{z}$ ), as one of the goals, in this paper [8]. The control system of the robotic arm uses this information to determine the next motion of the arm.

The core of the introduced solution is the task of estimating the position of the centre of gravity of an object in 3D space. By using step sequencing together with the help of two CCD camera systems precise methodological verification of measurements were reached. As key parameters of the vision systems (OMRON F150-3) are considered resolution $(512 \times 484)$, field of view (in our case $=0$ because were not used additional source of light) and focus $(35 \mathrm{~mm})$.

Through the use of auxiliary light sources in this technical solution it is possible to sense an object with minimum rectangular envelope cross section of 50x50mm from a distance up to $76 \mathrm{~mm}$. Experimental setup of the measuring stand requires the implementation of complex control, input - output and communication peripherals, mainly: control system for postprocessing, CCD camera systems with $35 \mathrm{mmm}$ lens, console, monitor and corresponding cables. The CCD cameras are directly fixed to an aluminium frame of modular construction, see figure 2 .

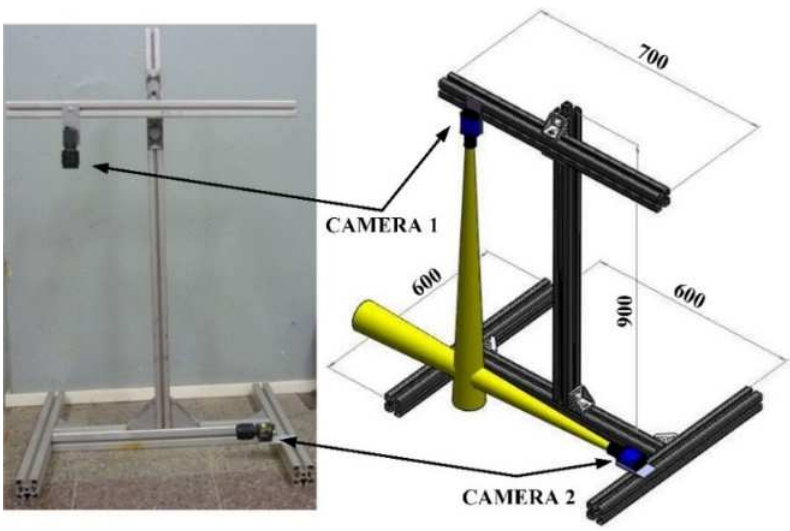

Figure 2 Realized technical solution - measurement experimental stand with CCD cameras

The outlined technical solution utilizes two CCD cameras mounted as seen in Figure 2 in order to be able to capture all object data coordinates [9]. This way, we try to avoid camera placement, which would not yield enough , z" coordinate axis data (shape, depth, high of the sensing object).

With respect to this requirement, the CCD cameras have been mounted in a coplanar fashion with the axis of the lenses perpendicular to one another and pointing 
towards the same point. The vertical CCD camera system is determining the object parameters in , $\mathrm{x}^{\prime \prime}$ and ,,y“ axis. Coordinate ,, $\mathrm{Z}^{\prime \prime}$, as the third supplementary axis, will be evaluated with the help of the horizontal camera system.

\subsection{Necessary steps for acquiring the coordinates of the scanned object}

The purpose of the following steps is to describe and present all necessary activities and functions needed for successful measurement. Firstly, both CCD camera systems need to undergo initial calibration, as is described in figure 3. This step contains several sub-activities, for example vision system start-up followed by CCD camera system registration and specification of additional light sources [10].

After these sub-activities it is necessary to set up and specify a processing mode type that is required for the next processing. Depending on the current lighting conditions the exposition time of the CCD camera needs to be adjusted.

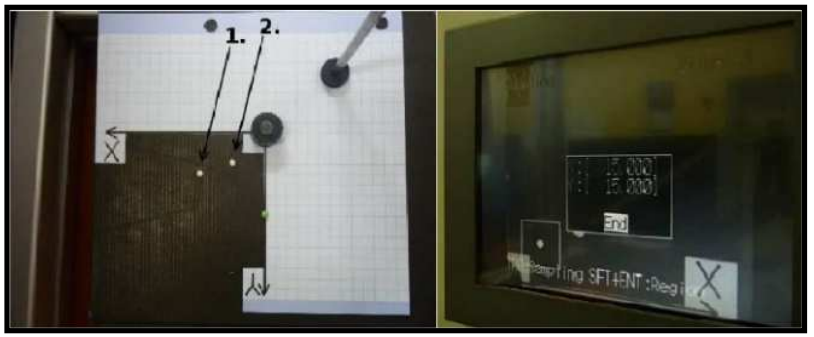

Figure 3 Initial calibration of CCD cameras

With the help of an accurate digital measurement instrument, the coordinates for the first calibration point were determined as $[\mathrm{x}=25 \mathrm{~mm}, \mathrm{y}=10 \mathrm{~mm}, \mathrm{z}=15 \mathrm{~mm}]$ and for the second calibration point as $[\mathrm{x}=30 \mathrm{~mm}, \mathrm{y}=15 \mathrm{~mm}$, $\mathrm{z}=15 \mathrm{~mm}]$. Two calibration points are sufficient for calibration because the magnification scale in axes , $\mathrm{X}^{\text {“ }}$ and „,y" is the same. An additional step of CCD camera system setup is object presence detection (if it is in the working envelope for both CCD cameras), see figure 4 .

Density averaging function (that is included in the vision system) was used on the measurement area. Basically, this function compares the two pictures brightness and evaluates them based on their grey scale [11].

Following this, an overall brightness average is determined with the measurement (firstly for the empty area, second for the area with sensed object).

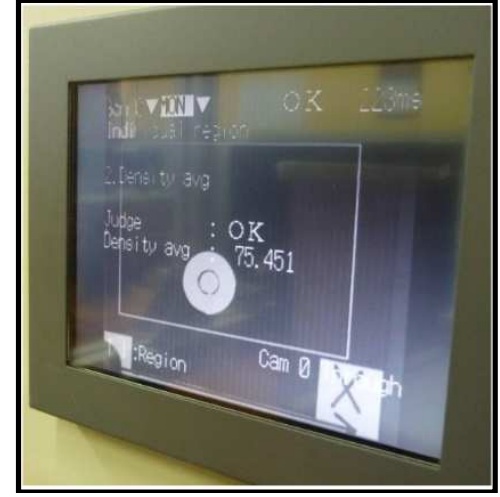

Figure 4 Detection of scanned object presence

The result of the verification process of the sensed object, in regard to the relevant lighting conditions, is the evaluation of the overall pixel density. The average density value of measured area was 74.451. In order the measurement to be correct it is necessary to set colour black/white colour threshold bounding conditions.

The next step is the identification of coordinate axes „, $\mathrm{x}$ “ and , $\mathrm{y}$ “ for the sensing object by using the gravity and area function. Following this the area of the sensed object is calculated as is viewed from the top. Sensed object is represented by white pixels and the remaining area is black, see figure 5 .

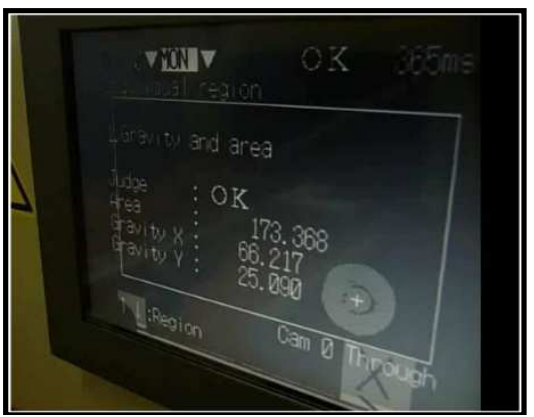

Figure 5 Determination of “ $x$ ” and " $y$ ” coordinates

Next step consists of determining the parameters tied to the , $\mathrm{Z}$ “ axis like the shape, depth and high of the sensed object, see figure 6. A sub-activity of this step consists of post-processing applied to the captured picture from vision system in a way that the edge position function was successfully applied to the sensed object. This function is characterized by the ability to detect the edges of the sensing object, provided there is sufficient contrast between the object and the environment. By applying this process, we can evaluate (in the appropriate direction) edges of the sensed object with respect to the simultaneously used functions "light to dark" (if is the sensed object is dark and the background is bright) or "dark to light" (if is the sensed object is bright and background is dark). 


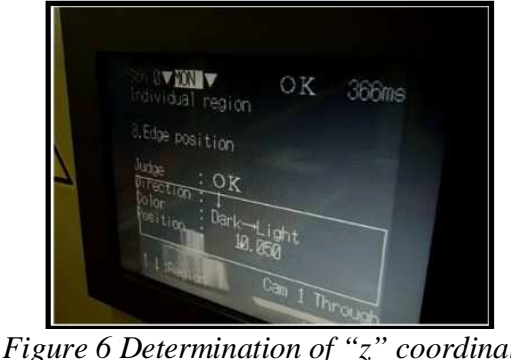

The last step consists of the determination of coordinates of the centre of gravity of the sensed object, which we determine by a simple mathematical operation $[\mathrm{x}, \mathrm{y}, \mathrm{z} / 2]$ - because the centre of gravity is determined at the middle of the object. Information that was obtained by this principle is then transferred into the computer via RS232C serial interface for advanced processing (coordinates transformation from external coordinate system to the coordinates of robotic arm control system).

Relevant information is sent directly to the control system of the robotic arm. We can state that the lighting conditions (light uniformity) and vertical camera system distance from the sensed object have majority influence on the results.

\section{Conclusion}

Existing classical approaches and techniques for determination of some necessary sensing object characteristic do not meet relevant needs of the automotive engineering industry and end customer needs. 3D vision sensors, as the main representative of vision systems, are an important key device for the automated assembly workplaces in order to enlargement its peripheral abilities and possibilities in the sense of industry 4.0 concept.

\section{Acknowledgement}

This work has been supported by the Slovak Grant VEGA 1/0330/19 - Research and design of algorithms and systems for the fusion of heterogeneous data in multisensor architectures and Slovak Grant VEGA 1/0389/18 Research and development of kinematically redundant mechanisms.

\section{References}

[1] KONIAR, D., HARGAŠ, L., LONCOVÁ, Z., SIMONOVÁ, A., DUCHOŇ, F., BEŇO, P.: Visual system-based object tracking using image segmentation for biomedical applications, Electrical Engineering, Vol. 99, pp. 1349-1366, 2017. https://doi.org/10.1007/s00202-017-0609-0
[2] TREBUŇA, F., HUŇADY, R., BOBOVSKÝ, Z., HAGARA, M.: Results and Experiences from the Application of Digital Image Correlation in Operational Modal Analysis, Acta Polytechnica Hungarica, Vol. 10, No. 5, pp. 159-174, 2013.

[3] SÁGA, M., BULEJ, V., ČUBOŇOVA, N., KURIC, I., VIRGALA, I., EBERTH, M.: Case study: Performance analysis and development of robotized screwing application with integrated vision sensing system for automotive industry, International Journal of Advanced Robotic Systems, Vol. 17, No. 3, pp. 1-23, 2020.

[4] DISTANTE, A., DISTANTE, C.: Handbook of Image Processing and Computer Vision, Springer Nature: Berlin, 2020.

[5] HASEGAWA, Y., NOF, S.Y.: Springer Handbook of Automation, Springer - Verlag, Berlin, 2020.

[6] VAGAŠ, M., GALAJdOVÁ, A., DŽONGOV, M.: Proposal of a vision system for automated line MPS 500, Technical Sciences and Technologies, Vol. 18, No. 4, pp. 108-113, 2019.

[7] SAUKKORIIPI, J., HEIKKILÄ, T., AHOLA, J.M., SEPPÄLÄ, T., ISTO, P.: Programming and control for skill-based robots, Open Engineering, Vol. 10, pp. 368376, 2020.

[8] CHEN, J., JING, L., HONG, T., LIU, H., GLOWACZ, A.: Research on a Sliding Detection Method for an Elevator Traction Wheel Based on Machine Vision, Symmetry, Vol. 12, pp. 1-14, 2020.

[9] SUKOP, M., HAJDUK, M., BALÁŽ, V., SEMJON, J., VAGAŠ, M.: Increasing degree of automation of production systems based on intelligent manipulation, Acta Mechanica Slovaca, Vol. 15, No. 4, pp. 58-63, 2011.

[10] TANNOURY, A., DARAZI, R., MAKHOUL, A., GUYEUX, CH.: Wireless multimedia sensor network deployment for disparity map calculation, IEEE Middle East and North Africa Communications Conference (MENACOMM), 2018.

[11] MOHAMED, A., CULVERHOUSE, P., CANGELOSI, A., YANG, CH.: Active stereo platform: online epipolar geometry update, EURASIP Journal on Image and Video Processing, Vol. 54, 2018.

\section{Review process}

Single-blind peer review process. 